\title{
CARACTERISTICAS DE LA INFESTACIÓN DE Diplectanum sp. (MONOGENEA: MONOPISTHOCOTYLEA: DIPLECTANIDAE) EN EL AYANQUE Cynoscion analis Jenyns (PISCES: TELEOSTEI: SCIAENIDAE) \\ CHARACTERISTICS OF INFECTION OF Diplectanum sp. (MONOGENEA: MONOPISTHOCOTYLEA: DIPLECTANIDAE) IN Cynoscion analis Jenyns (PISCES: TELEOSTEI: SCIAENIDAE)
}

\author{
José Iannacone", Wilfredo Mejía, Francisco Alcócer, \\ Gian Briones y Amid Román
}

\section{RESUMEN}

120 especimenes de "Ayanque" Cynoscion analis Jenyns fueron colectados del Terminal Pesquero de Chorrillos, Lima. Perú, entre enero y febrero del 2000 y necropsiados para estudiar el monogeneo ectoparásito Diplectanum $s p$. a nivel de los filamentos branquiales. Este parásito es considerado una especie central, por parasitar a más de $2 / 3$ de los hospederos examinados. El número de peces hembras fue de 80 ; mientras que el de machos, 40 . La longitud de las hembras estuvo entre $16,4-27,0 \mathrm{~cm}$ (Promedio $=21,08 \pm 2,24 \mathrm{~cm}$ ). La longitud de los machos estuvo entre $16,5-26,3 \mathrm{~cm}$ (Promedio $=20,47 \pm 2,06$ ). La prevalencia de infestación de Diplectanum fue de $72,5 \%$, la intensidad media fue $3,16 \pm 2,84$ y la abundancia media fue $2,29 \pm 2,80$. Este monogeneo mostró una distribución espacial sobredispersa $(2,55)$ a nivel de los peces hospederos. Se observó una correlación entre la intensidad media de intestación y la longitud estandar de $C$. analis. Los peces machos $(4,14 \pm 3,75)$ presentaron significativamente una mayor intensidad media que las hembras $(2,67 \pm 2,14)$. Se observó una mayor prevalencia, intensidad y abundancia media de Diplectanum en el ll arco branquial. Sin embargo, no se encontró ninguna preferencia al dividir cada uno de los arcos en ires sectores (anterior, medio y posterior respectivamente). Finalmente se comparan los resultados obtenidos con la estructura poblacional de otros monogeneos ectoparásitos en peces marinos. Diplectanum sp. es un nuevo registro para el Perú para $C$. analis.

Palabras clave: Ayanque, Cynoscion analis, Monogenea, Diplectanum, parásitos

\section{ABSTRACT}

1:0 Cynoscion analis Jenyns were collected from Chorrillos fishmarket, Lima, Perú, between January and February 2000 and necropsied 10 study ectoparasite monogenean Diplectanum sp. from branchial filaments. This parasite was considered a core specie, because it parasites $2 / 3$ hosts examined. Of the fishes collected 80 were female and 40 male. Females and males showed a standard length between $16,4-27,0 \mathrm{~cm}(\mathrm{mean}=21,08 \pm 2,24)$ and $16,5-26,3 \mathrm{~cm}$ (mean $=20,47 \pm 2,06$ ), respect.vely. The prevalence of infection of Diplectanum was $72,5 \%$, mean intensity and abundance were $3,16 \pm 2,84$ and $2,29 \pm 2,80$ respectively. This monogenean showed an overdispersal spatial distribution $(2,55)$ at level of fish hosts. We observed a correlation between mean intensity of infestation and standard length of $C$. analis. Males $(4,14 \pm 3,75)$ showed a higher mean intensity than females $(2,67 \pm 2,14)$. A high prevalence, intensity and abundance of Diplectanum to II gill was found. However, we did not find neither preference when we separated each gill in three parts (fore, middle and hind respectively). Finally, we coinpared our results of population assemblages of other ectoparasite monogeneans in marine fishes. Diplectanum sp. is a new record to $C$, analis and to Peru.

Key words: Cynoscion analls, Monogenean, Diplectanum, parasites.

\section{INTRODUCCIÓN}

La diversidad biológica del ambiente marino ha recibido poca atención, no obstante presentar sistemas tan diversos y frágiles comparados a los del bosque tropical lluvioso

\footnotetext{
Laboratorio de Ecofisiología. Facultad de Ciencias Naturales y Maremálicas. Unversidad Nacional Federico Viliameal. Calle San Marcos 383, Pueblo Libre. Lima. Poru. Telefax: 4600930 . E-mail: Joselorena terre.com pe
}

(Tarazona y Valle, 1998). El empleo de las branquias y áreas operculares de los peces para el análisis de sus ectoparásitos tiene varias ventajas, ya que pudieran ser considerados hábitats relativamente pequeños que pueden ser examinados en poco tiempo, y se pueden mapear con facilidad y exactitud (Rohde, 1991; Rohde et al., 1994; Luque, 1996; Lo, 1999; Oliva, 1999). 
En los últimos años, se han publicado varios trabajos sobre la fauna parasitaria de peces de la familia Sciaenidae (Osteichthyes) de la Costa Peruana como Stellifer minor (Tschudi), Paralonchurus peruanus (Steindachner), Sciaena deliciosa (Tschudi), Sciaena fasciata (Tschudi) y Menticirrhus ophicephalus (Jenyns), estableciendo patrones básicos de las infracomunidades parasitarias en relación al tamaño de los hospedadores, sexo, variación estacional y estructura de las comunidades parasitarias (Oliva et al., 1989, 1990; Farfán 1990; Iannacone 1991; Luque y Oliva, 1993; Luque 1994b, 1996; Iannacone et al., 1997). En las comunidades de ectoparásitos de la costa peruana se ha encontrado una alta predominancia de los monogeneos diplectánidos y dactylogíridos (Luque 1994. 1996).

Cynoscion analis es un sciénido de importancia en las cadenas tróficas marinas. Su distribución geográfica conocida va desde Perú a Chile (Chirichigno, 1974; Mendo et al. 1988). C. analis ha sido incompletamente evaluado con relación a su fauna parasitaria (Saldarriaga 1977; Luque et al., 1991).

Este trabajo representa un primer análisis cuantitativo del monopistocotylea Diplectanum sp., metazoario ectoparásito de $C$. analis, con el objetivo de evaluar la influencia del tamaño y sexo del hospedero, sobre la prevalencia, intensidad y abundancia media de este monogeneo, así como su distribución a nivel branquial.

\section{MATERIAL Y MÉTODOS}

\section{Obtención del material biológico}

Se adquirieron 120 especímenes de "Ayanque", $C$. analis entre enero y febrero del 2000 en el Terminal Pesquero de Chorrillos, Perú (12" $30 \mathrm{~S}$, 76" $50 \mathrm{E}$ ). Los peces se llevaron al laboratorio tratando de que se mantuvieran en óptimo estado de conservación. Diplectanum sp. fue clasificado de acuerdo a las claves taxonómicas de Oliver (1982). Estos se colectaron de los arcos branquiales que fueron contabilizados el mismo día de su colecta, para evitar así el deterioro de gran parte de la población parasitaria por efecto de la alta temperatura propia de la estación de verano. Una vez en el laboratorio, se procedió primero a la extracción de los opérculos, luego a los arcos branquiales en orden, examinando su cara interna y de uno en uno, los que fueron divididos en tres secciones $a, b, c$ (anterior, medio y posterior respectivamente) y fueron colocados en una placa Petri con la cantidad necesaria de solución salina al $0,85 \%$ para facilitar el desprendimiento de la mucosidad y de los Diplectanum. Todo esto se llevó a cabo con la ayuda de un microscopio compuesto y de un microscopio estereoscopio, siguiendo las recomendaciones de Rohde (1980). Este monogeneo presentó una longitud total corporal de 895 (810-990) $\mu$ y un ancho corporal de 105 (85-112) $\mu(n=6)$.

Se determinó en los hospederos el sexo y la longitud estándar (en $\mathrm{cm}$ ). Además se registró el número y lugar de infestación del Diplectanum por pez hospedero (arcos branquiales y su respectiva sección).

Identificados estos ectoparásitos y previamente limpiados se procedió a su coloración con carmín acético Semichon's diluido con alcohol al $70 \%$ (6:1) durante unos 5 minutos. Luego se sometió a una batería de alcoholes $(70,85,95$ y 99\%). Finalmente este monogeneo fue montado en medio de Bálsamo de Canadá.

\section{Estructura Poblacional}

Se determinó la Prevalencia (PREV), Intensidad Media (IM) y Abundancia Media (AM) por pez, por lado derecho e izquierdo y para cada uno de los arcos branquiales con 
sus respectivas secciones. La longitud estándar de los hospederos se dividió en seis rangos de $1,8 \mathrm{~cm}$ cida uno. El número de peces hembras fue de 80 ; mientras que el de machos, 40. Las hembras presentaron una longitud entre $16,4-27,0 \mathrm{~cm}$ (promedio $=21,08 \pm 2,24 \mathrm{~cm}$ ). Los machos presentaron una longitud entre $16,5-26,3 \mathrm{~cm}$ (promedio $=20,47 \pm 2,06 \mathrm{~cm}$ ). El Coeficiente de Dispersión (CD) empleado fue determinado como una relación entre la Varianza/ IM de Diplecianum.

La prueba de $t$ de student, previa evaluación de homogeneidad de varianzas empleando la prueba de Levene, fue usada para determinar si la longitud estándar de los peces hospederos machos y hembras presentaban diferencias significativas. La influencia de la talla del hospedero en la PREV de infestación de Diplectanum $s p$. se determinó usando el Coeficiente de Correlación de Pearson (r). Este se utilizó nuevamente para determinar la relación del tamaño del hospedero con la IM y AM del Diplectanum sp. Se aplicó el $\chi^{2}$ para tablas de contingencia para determinar el grado de dependencia entre el sexo del hospedero y la PREV de este ectoparásito. El efecto del sexo en la IM y la AM de infestación de Diplectanum sp. se calculó utilizando la prueba de $\mathrm{t}$ de student, previo empleo de la prueba de Levene. Se utilizó nuevamente la prueba de $\chi^{2}$ para tablas de contingencia para determinar si había preferencias en la PREV de Diplectanum para ciertos arcos y secciones branquiales en el pez hospedero. Para el cálculo de la preferencia con relación a la IM y AM en los arcos y secciones branquiales se uso el ANDEVA de una vía con un diseño completamente aleatorizado; en el caso de existir diferencias significativas, se utilizó la prueba de Tukey para poder notar el o los posibles arcos y sectores branquiales que estuvieran causando las diferencias (Zar, 1996). Se usó nuevamente la prueba de $t$ de student para determinar si existen diferencias entre la PREV e IM entre monogeneos monopistocotylea y poliopistocotylea parásitos en peces marinos peruanos, respectivamente. Además se determinó la correlación (r) de Pearson existente de PREV y la IM de todos los monogeneos evaluados (Rohde et al., 1995). El nivel de significancia fue evaluado a $\alpha=0,05$.

La terminología ecológica para la PREV, IM y AM siguió los criterios de Bush et al. (1990). Se usó la terminología de especie central propuesta por Bush y Holmes (1986) Para el cálculo de las pruebas estadísticas descriptivas se usó el paquete estadístico SPSS 9.0 para Windows 98.

\section{RESULTADOS}

La Tabla 1 muestra 'a prevalencia, intensidad y abundancia media de infestación de Diplectanum en los 120 hospederos muestreados. Además se observa una

Tabla 1. Microhabitat, prevalencia (PREV), intensidad (IM) y abundancia media (AM) y coeficiente de dispersión (CD) de Diplectanum sp. en 120 especímenes de $C$. analis procedentes del Terminal Pesquero de Chorrillos, Lima, Perú.

\begin{tabular}{ll}
\hline Ectoparásito & Diplectanum sp. \\
\hline Microhábitat & Filamentos branquiales \\
\hline PREV & $72,5 \%$ \\
IM & $3,16 \pm 2,84$ \\
AM & $2,29 \pm 2,80$ \\
CD & 2,55 \\
\hline
\end{tabular}


distribución sobredispersa o contagiosa, pues el coeficiente de dispersión es mayor a $1(2,55)$ y finalmente se observa su preferencia al microhábitat de los filamentos branquiales.

El promedio de la longitud de los peces machos (promedio $=20,47 \pm 2,06 \mathrm{~cm}$ ) y de las hembras (promedio $=21,08 \pm 2,24 \mathrm{~cm}$ ) en la muestra analizada, asumiendo igualdad de varianzas, no fue significativamente diferente $(t=1,23, \mathrm{P}=0,22)$.

La PREV y la AM de infestación de Diplectanum no mostraron ninguna correlación significativa con la longiiud estándar de $C$. analis $(r=0,19 ; P=0,71)$ y ( $r=0,13 ; P=0,15$ ) respectivamente. En cambio, la IM y la longitud estándar sí presentaron una correlación positiva y significativa $(r=0,23 ; P=0,03)$.

Con relación al sexo de $C$. analis, los individuos machos presentaron una IM de

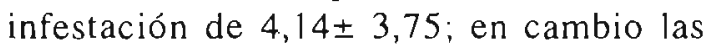

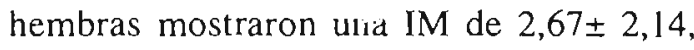
observándose diferencias estadísticamente significativas, pero asumiendo desigualdad de varianzas por la prueba de Ltevene $(t=1,95$; $\mathrm{P}=0,05)$. Con respecto a la $\mathrm{AM}$ versus el sexo del pez, las hembras presentaron un valor de 3,0 $\pm 3,69$ y los machos de 4,14 $\pm 3,75$, y no se observó diferencias significativas $(t=1,68 ; \mathrm{P}=0,09)$.

La PREV de Diplectanum y el sexo del hospedero no indic 6 diferencias significativas entre los machos y las hembras $\left(\chi^{2}=0,10 ; P>\right.$ $0,05)$.

Diplectanum mostró para la PREV preferencias por el II arco branquial $(42,5 \%)$ en $C$. analis, $\left(\chi^{2}=9,98 ; P<0,05\right)$, seguido por el III $\operatorname{arco}(37,5 \%)$, I $\operatorname{arco}(31,66 \%)$ y el IV arco $(24,2 \%)$. El mismo comportamiento se observó al analizar los arcos branquiales derechos $\left(\chi^{2}=8,46 ; P<0,05\right)$ e izquierdos $\left(\chi^{2}=10,1 ; \mathrm{P}<0,05\right.$ ) (Tabla 2). No se observó preferencias en la PREV de Diplectanum por

Tabla 2. Prevalencia (PREV) de infestación Diplectanum sp. en 120 C. analis procedentes del Terminal Pesquero de Chorrillos, Lima, Perú.

\begin{tabular}{|c|c|c|c|c|c|c|c|c|c|}
\hline & \multicolumn{4}{|c|}{$\begin{array}{l}\text { Área Derecha } \\
54,17 \%\end{array}$} & \multicolumn{4}{|c|}{$\begin{array}{l}\text { Área Ixquierda } \\
50 \%\end{array}$} & \\
\hline & a & b & c & $t$ & a & b & c & $t$ & $T$ \\
\hline I & $5,83 \%$ & $7.5 \%$ & $7,5 \%$ & $20,83 \%$ & $5 \%$ & $5,3 \%$ & $9,17 \%$ & $17,5 \%$ & $31,66 \%$ \\
\hline II & $10,83 \%$ & $9,17 \%$ & $10 \%$ & $25,83 \%$ & $15 \%$ & $12,5 \%$ & $8.3 .3 \%$ & $30 \%$ & $42,5 \%$ \\
\hline Ш & $7,5 \%$ & $11,67 \%$ & $8,33 \%$ & $23,33 \%$ & $5,83 \%$ & $12,5 \%$ & $6,67 \%$ & $20,83 \%$ & $37,5 \%$ \\
\hline $\mathrm{N}$ & $6,67 \%$ & $4,17 \%$ & $5 \%$ & $15 \%$ & $4,16 \%$ & $6,67 \%$ & $4,17 \%$ & $14,17 \%$ & $24,17 \%$ \\
\hline$t$ & $25,83 \%$ & $26,67 \%$ & $24,17 \%$ & & $24,17 \%$ & $30,83 \%$ & $22,5 \%$ & & \\
\hline
\end{tabular}

$a=$ Sector anterior del arco branquial

$\mathrm{b}=$ Sector medio del arco branquial.

$c=$ Sector posterior del arco branquial.

$\mathrm{t}=$ Total para arcos branquiales derechos o izquierdos

$\mathrm{T}=$ Total de ambos arcos branquiales.

I, II, III y IV = Arcos branquiales I, II, III y IV. 
ninguna de las 24 secciones branquiales $\left(\chi^{2}=5,87 ; P>0,05\right)$.

El análisis de varianza realizado con relación a la IM y a AM en los cuatro arcos branquiales para Diplecıanum indicó el mismo comportamiento que para la $\operatorname{PREV}(F=4,39$; $\mathrm{P}=0,005)$ y $(F=4,58 ; \mathrm{P}<0,05)$ respectivamente (Tablas 3 y 4 ). Sin embargo, no se observaron preferencias en la IM y AM para ninguna de las 24 secciones branquiales $(F=1,09 ; P>0,05)$ y $(F=1,57 ; P=0,22)$, respectivamente.

La Tabla 5 señala valores comparativos de PREV e IM de infestación de diferentes monogeneos monopistocotylea y poliopistocotylea principalmente de la costa peruana. El análisis comparativo de la PREV

Tabla 3. Intensidad media (IM) de infestación Diplectanum sp. en 120 C. analis procedentes del Terminal Pesquero de Chorrillos, Lima, Perú.

\begin{tabular}{|c|c|c|c|c|c|c|c|c|}
\hline \multicolumn{4}{|c|}{$\begin{array}{l}\text { Área Derecha } \\
2,06 \pm 1,39\end{array}$} & \multicolumn{4}{|c|}{$\begin{array}{l}\text { Área Izquierda } \\
2,33 \pm 1,85 \\
\end{array}$} & \multirow[b]{2}{*}{$\mathbf{T}$} \\
\hline a & $\mathbf{b}$ & c & $\mathbf{t}$ & a & b & c & $\mathbf{t}$ & \\
\hline 1 & $1,44 \pm 0,73$ & 1 & $1,16 \pm 0,47$ & 1 & $1,43 \pm 0,53$ & $1,45 \pm 0,69$ & $1,52 \pm 0,93$ & $1,61 \pm 1,17$ \\
\hline $1,15 \pm 0,55$ & $1,09 \pm 0,30$ & $1,17 \pm 0,58$ & $1,32 \pm 0,70$ & $1,22 \pm 0,43$ & $1,53 \pm 0,74$ & $1,1 \pm 0,32$ & $1,56 \pm 0,77$ & $1,94 \pm 1,19$ \\
\hline $1,22 \pm 0,44$ & $1,14 \pm 0,36$ & $1,4 \pm 0,84$ & $1,46 \pm 0,79$ & I & $1,13 \pm 0,35$ & $1,12 \pm 0,54$ & $1,28 \pm 0,61$ & $1,64 \pm 0,98$ \\
\hline $1,12 \pm 0,35$ & I & $1,5 \pm 0,55$ & $1,28 \pm 0,57$ & l & 1 & $1,2 \pm 0,45$ & $1,12 \pm 0,33$ & $1,45 \pm 0,87$ \\
\hline $1,35 \pm 0,62$ & $1,44 \pm 0,88$ & $1,59 \pm 1,24$ & & $1,38 \pm 0,68$ & $1,57 \pm 0,96$ & $1,56 \pm 0,70$ & & \\
\hline
\end{tabular}

$a=$ Sector anterior del arco branquial

$b=$ Sector medio del arco branquial.

$c=$ Secior posierior del arco branquial.

$t=$ Tolal para arcos branquiales derechos o izquierdos.

$T=$ Toral de ambos arcos branquiales.

I, II, III y IV = Arcos branquiales I. II, III y IV.

Tabla 4. Abundancia media (AM) de infestación Diplectanum sp. en 120 C. analis procedentes del Terminal Pesquero de Chorrillos, Lima, Perú.

\begin{tabular}{cccc|cccc|c}
\hline \multicolumn{4}{c|}{$\begin{array}{c}\text { Area Derecha } \\
1,12 \pm 1,45\end{array}$} & \multicolumn{5}{c|}{$\begin{array}{c}\text { Área Izquierda } \\
1,17 \pm 1,75\end{array}$} \\
\hline $\mathbf{a}$ & $\mathbf{b}$ & $\mathbf{c}$ & $\mathbf{t}$ & $\mathbf{a}$ & $\mathbf{b}$ & $\mathbf{c}$ & $\mathbf{t}$ & $\mathbf{T}$ \\
$0,06 \pm 0,24$ & $0,11 \pm 0,43$ & $0,08 \pm 0,26$ & $0,24 \pm 0,52$ & $0,05 \pm 0,22$ & $0,08 \pm 0,36$ & $0,13 \pm 0,45$ & $0,27 \pm 0,69$ & $0,51 \pm 1$ \\
$0,03 \pm 0,40$ & $0,10 \pm 0,33$ & $0,12 \pm 0,39$ & $0,34 \pm 0,68$ & $0,18 \pm 0,47$ & $0,19 \pm 0,57$ & $0,09 \pm 0,32$ & $0,47 \pm 0,83$ & $0,82 \pm 1,23$ \\
$0,09 \pm 0,34$ & $0,13 \pm 0,39$ & $0,12 \pm 0,45$ & $0,34 \pm 0,73$ & $0,06 \pm 0,24$ & $0,14 \pm 0,40$ & $0,08 \pm 0,30$ & $0,27 \pm 0,59$ & $0,62 \pm 1$ \\
$0,08 \pm 0,30$ & $0,04 \pm 0,20$ & $0,08 \pm 0,35$ & $0,19 \pm 0,51$ & $0,04 \pm 0,20$ & $0,07 \pm 0,25$ & $0,05 \pm 0,25$ & $0,16 \pm 0,41$ & $0,35 \pm 0,75$ \\
$0,35 \pm 0,67$ & $0,38 \pm 0,78$ & $0,38 \pm 0,91$ & & $0,33 \pm 0,68$ & $0,48 \pm 0,90$ & $0,35 \pm 0,73$ & & \\
\hline
\end{tabular}

$a=$ Sector anterior del arco branquial.

$\mathrm{b}=$ Sector medio del arco branquial.

$\mathrm{c}=$ Sector posterior del arco branquial.

$t=$ Total para arcos branquiales derechos o izquierdos.

$\mathrm{T}=$ Tolal de ambos arcos branquiales.

I, II, III y IV = Arcos branquiales I, II, III y IV. 


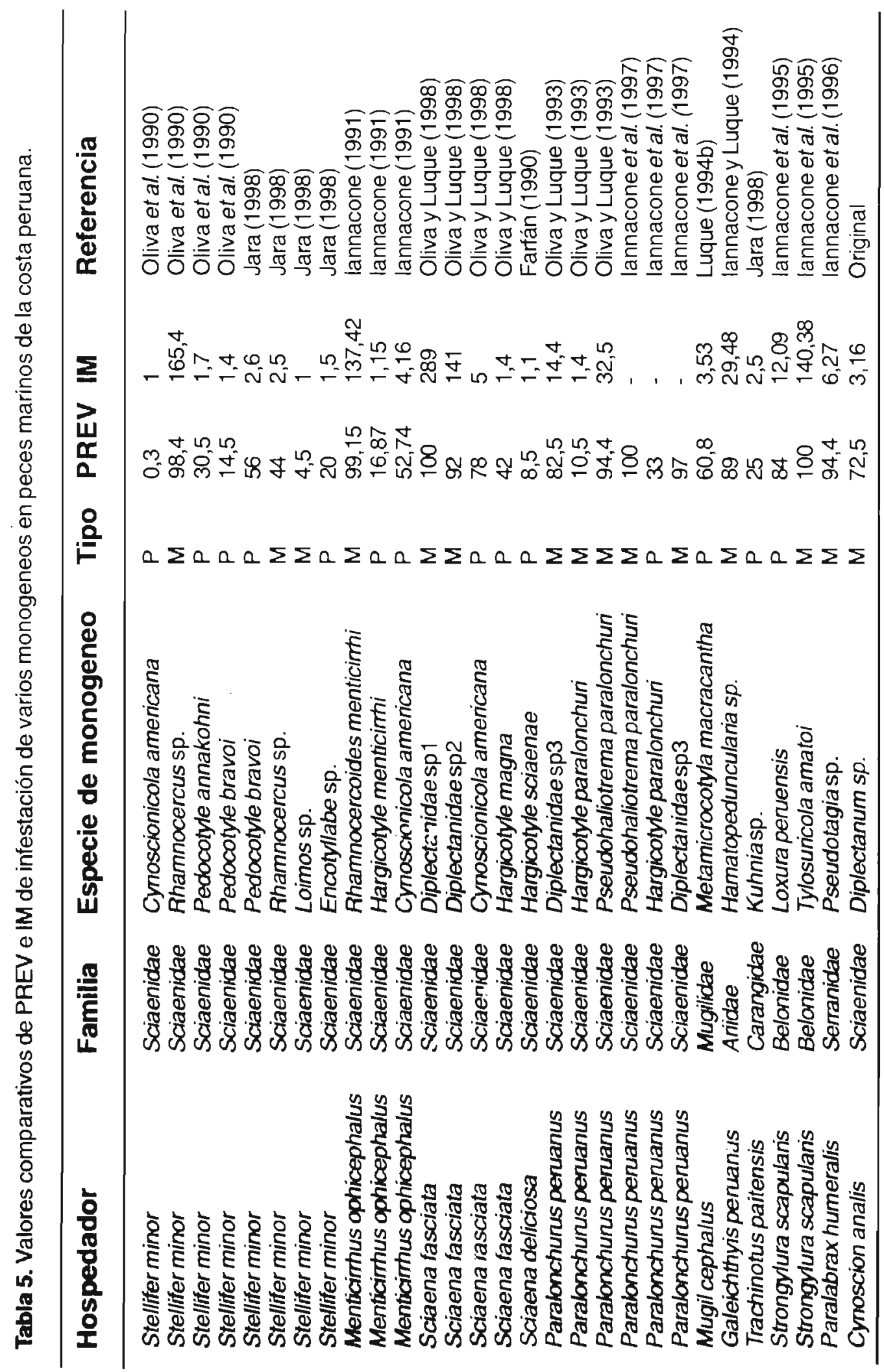


los monopistocotylea (promedio $=78,55 \pm$ $32,39 \%$ ) y los poliopistocotylea (promedio $=37,30 \pm 25,79 \%$ ), mediante la prueba de $\iota$ de student mostró diferencias significativas $(t=3,77 ; \mathrm{P}=0,001)$. El mismo comportamiento se observó para la IM de los monopistocotylea (promedio $=74,13 \pm 91,31$ ) y los poliopistocotylea (promedio $=3,01 \pm 3,01$ ) $(t=2,8 l ; \mathrm{P}=0,01)$. La PREV y la IM estuvieron altamente correlacionados $(r=0,603 ; P=0,001)$.

\section{DISCUSIÓN}

Saldarriaga (1977) y Luque et al. (1991) citaron cuatro parásitos para $C$. analis, a los monogeneos Neoheterobothrium cynoscioni (Mac Callum, 1917), Cynoscionicola cynoscioni; Tantaleán, Martínez y Escalante (1987), a las larvas de céstodo Otobothrium sp. y al acantocéfalo Tegorhynchus sp.

Las características poblacionales de los procesos infecciosos originados por Diplectanum sp. en $C$. analis están en concordancia con los cuadros descritos para varios sistemas pez marino-monogeneo monopistocotylea y en particular con la información disponible para las comunidades parasitarias de otras especies de esciénidos ( $P$. peruanus, $S$. minor, $M$. ophicephalus, $S$. fasciuta) (Iannacone, 1991; Luque y Oliva, 1993; Luque 1994a, 1996; Oliva y Luque, 1998). Oliva y Luque (1998) señalan que la principal característica de las comunidades parasitarias de los peces esciénidos es la fuerte predominancia de monogeneos ectoparásitos poilonchoineos-monopistocolylea, a nivel cualitativo $(75,6 \%)$ y cuantitativo $(94,4 \%)$.

Estos grupos de monogeneos (diplectánidos y dactylogíridos) muestran ciclos biológicos de corta duración, tal como fue comprobado por Kingston et al. (1969) y Silan y Maillard (1989), lo que posiblemente ocasione sus altas prevalencias. Jara (1998) muestra que el parásito microscópico Rhamnocercus sp., parasito de $S$. minor, se reproduce más rápidamente y por ello se les encuentra generalmente en porcentajes altos, a lo cual contribuye el hecho de que muchas veces los huevos quedan atrapados en las mucosas tanto de las branquias como del integumento permitiendo sobre el mismo hospedador las salidas de las larvas oncomiracidias y la continua reinfección. Según la clasificación de los parásitos platelmintos de Whittington (1997), son considerados especies con estrategia "r", por sus elevados potenciales reproductivos en sus peces hospederos.

Luque (1996) señala que los esciénidos conjuntamente con los serránidos son las familias de peces marinos por las cuales los diplectánidos demuestran mayor especificidad parasitaria. Las especies de diplectánidos son altamente específicos en la mayoría de sus hospederos (Hayward, 1997).

La distribución sobredispersa o contagiosa es considerada una regla en los procesos parasitarios y es originada por la heterogeneidad del comportamiento del hospedador, por modelos de agregación espacial de los estados infectivos, diferencias en la susceptibilidad o habilidad de defensa del hospedero y a características alimentarias individuales (Anderson y Gordon, 1982; Iannacone, 1991). Estos causales podrían explicar el comportamiento de Diplectanum sp. de $C$. analis.

Otros monogeneos polionchoineos como Rhamnocercoides menticirrhi )Luque y Iannacone 1991), ectoparásito de $M$. ophicephalus en la Costa Central del Perú, mostraron un coeficiente de dispersión mucho más alto (13,65); lo mismo con Rhamnocercus sp. en $S$. minor $(56,6)$, Hamatopeduncularia sp. en Galeichthyis peruanus (L.) (7,61), oligonchoineos como Metamicrocotyla macracantha Tantaleán 1974 en Mugil cephalus L. $(4,85)$, Loxura peruensis Oliva y Luque 1995 en Strongylura scapularis ( = Belone scapularis) (Jordan y Gilbert) $(4,1)$; Hargicotyle menticirrhi Oliva y Luque 
$1989(1,11)$ y en Cynoscionicola americana Tantalean, Martínez y Escalante, $1987(7,84)$ en $M$. ophicephalus (Oliva et al., 1990; Iannacone, 1991; Iannacone y Luque, 1994; Luque, 1994a,b; Iannacone et al., 1995). Nuestros resultados muestran un bajo CD para Diplectanum, en comparación con otros monogeneos en peces marinos de la costa peruana; esto pudiera deberse a que $C$. analis, como especie bentopelágica, según Rohde $e t$ al. (1995), presenta una menor PREV e IM que las especies propiamente bentónicas, siendo que la IM presenta una alta influencia en el valor del CD.

Hayward et al. (1998) señalan que la longitud del hospedero es un factor importante y determinante en la estructura poblacional de los ectoparásitos en peces. Oliva et al. (1990) señalaron que Rhamnocercus sp. parásito de las branquias de $S$. minor mostró una IM directamente correlacionada con la longitud del hospedador, explicando estos resultados en función de que los peces de mayor tamaño tienen más tiempo disponible para una infestación acumulada. $R$. menticirrhi en $M$. ophicephalus, presentaron el mismo comportamiento (Iannacone, 1991). En el presente estudio Diplectanum sp. mostró un comportamiento similar para la IM y la talla del hospedero. Ello podría explicarse por: (1) acumulación de Diplectanum en el tiempo, y (2) aumento progresivo del área disponible en las branquias al incrementarse la longitud de C. analis (Morand et al., 1999). Una mejor explicación de estos modelos solo será posible cuando los ciclos de vida de los parásitos y sus relaciones con la dinámica poblacional del hospedero lleguen a ser conocidas.

Luque y Oliva (1993) estudiando a Diplectanum sp. y a Pseudohaliotrema paralonchuri Luque y Iannacone 1989 en $P$. peruanus encontraron una ausencia de correlación entre la longitud del hospedador y la AM de ambos monogeneos parásitos, atribuyéndolo a una homogeneidad en el comportamiento del pez durante toda su vida, lo que permite reclutar uniformemente durante su evolución ontogénica las mismas especies de ectoparásitos con ciclos de vida directos. Este comportamiento fue observado para el caso de la AM de Diplectanum parásito de C. analis.

E] sexo de $C$. analis no demostró influencia sobre la infectación de Diplectanum, lo que también se observa en muchos sistemas pez-parásito (Lasse, 1989; Sutherland, 1989; Gonzales y Acuña, 1998; Sasal et al., 1999). Cezar y Luque (1999), indican que la carencia de correlación pudiera atribuirse a la similaridid en la relaciones ecológicas (comportamiento, hábitat y dieta) de los peces machos y hembras.

Numerosos estudios han demostrado que los parásitos están limitados en su distribución en el pez hospedador. Existen varios factores implicados: la especificidad morfológica del sustrato; la selección activa del microhábitat en función del patrón de las corrientes de agua en la cámara branquial; la concentración de especimenes para facilitar la c:spula y la modificación del hábitat por la presencia de otras especies para evitar procesos competitivos (Iannacone, 1991; Luque, 1994b). La forma de ingreso del parásito (pasivo o activo) influencia la preferencia por un determinado arco branquial, además de la influencia del volumen de ventilación y el patrón de corriente que se produce en la cavidad branquial (Gutiérrez y Martorelli, 1994). Luque (1994a) señaló que el grueso de la corriente de agua fluye sobre los $\operatorname{arcos} 2$ y 3. A pesar de esta influencia, muchas especies de parásitos han sufrido modificaciones de los órganos de fijación que les permiten ocupar cualquier hábitat (Rohde, 1982; Oliva et al., 1989). Hamatopeduncularia sp. prefiere e] I, II y III arco branquial (Iannacone y Luque, 1994), R. menticirrhi parasita preferencialmente el II arco branquial (Iannacone, 1991), lo que indicaría un posible ingreso pasivo (Davey, 1980). En nuestro caso, Diplectanum en $C$. analis prefiere el II y III 
arco branquial, tanto en PREV, IM y AM de infestación, al igual que $R$. menticirrhi, lo que indicaría un ingreso pasivo al hospedador.

Geets et al. (1997) señalan que en el pez marino Siganus autor, los monogeneos Pseudohaliotrema sp., Tetrancistrum sigani, Microcotyle mouwoi muestran preferencias a ciertos sectores branquiales de su pez hospedador; sin embargo, la presencia de alguna especie de parásito es independiente de la amplitud de nicho para estas cuatro diferentes especies de helmintos branquiales. Además indican que la alta distribución agregada de estos parásitos en los filamentos branquiales permitiría el contacto intraespecífico para cada una de estas especies (Janovy et al., 1991). El Hafidi et al. (1998) muestran para dos ectoparásitos de $M$. cephalus como Metamicrocotyla cephalus y Microcoryle mugilis preferencia por ciertos sectores a nivel de los arcos branquiales; sin embargo, estas dos especies no inducen cambios en su distribución a nivel de sus sectores preferidos, por el contrario ocurre un incremento de su prevalencia e intensidad de infestación en comparación con sus infecciones monoespecíficas. Nuestros resultados indican, a nivel de la PREV, IM y $\mathrm{AM}$, que no existe una preferencia por ninguno de los 24 sectores de los arcos branquiales (Tabla 2 y 3 ).

\section{AGRADECIMIENTOS}

Los autores expresan su agradecimiento al Laboratorio de Biología y Ecofisiología de la Facultad de Ciencias Naturales y Matemáticas de la Universidad Nacional Federico Villarreal por su apoyo a la presente investigación.

\section{LITERATURA CITADA}

Anderson, R. M. y D. M. Gordon, 1982. Processes influencing the distribution of parasite numbers within host populations with special emphasis on parasite-induced host mortality. Parasitology 85 : 373-398.

Bush, A. O. y J. C. Holmes. 1986. Intestinal helminthes of lesser scaup ducks: an interactive community. Can. J. Zool. 64: 142-152.

Bush, A. O.; K. D. Lafferty; J. L. Lotz y A. W. Shostak. 1997. Parasitology meets ecology on its own terms: Margolis et al. revisited. J. Parasitol. 83: 575-583.

Cezar, A. D. y J. L. Luque. 1999. Metazoan parasites of the Atlantic Spadefish Chaetodipterus faber (Teleostei: Ephippidae) from the Coastal Zone of the State of Rio de Janeiro, Brazil. J. Helminthol. Soc. Wash. 66: 14-20.

Chirichigno, N. 1974. Clave para identificar los peces marinos del Perú. Inf. Inst. Mar. Perú-Callao 44: 1-387.

Davey, J. T. 1980. Spatial distribution of the copepod Lernanthropus kroyeri on the gills of bass Dicentrachus labrax (L.). J. mar. biol. Ass. U.K. 60: 1 061- 1067.

El Hafidi, F.; O. Berrada-Rkhami; T. Bernazzou y C. Gabrion. 1998. Microhabitat distribution and coexistence of Microcotylidae (Monogenea) on the gills of the striped mullet Mugil cephalus: change or competition? Parasitol. Res. 84: 31 5-320.

Farfán, C. 1990. Aspectos ecológicos de la Fauna parasitaria (Metazoa) de Sciaena deliciosa (Tschudi, 1844) (Pisces: Teleostei). Tesis Lic. En Biología. Univ. Ricardo Palma, Lima, Perú. $49 \mathrm{pp}$.

Geets, A.; H. Coene y F. Ollevier. 1997. Ectoparasites of the whitespotted rabbitfish, Siganus autor (Valenciennes, 1835) off the Kenyan Coast: distribution within the host population and site selection on the gills. Parasitology 115:69-79.

González, M.T. y E. Acuña. 1998. Metazoan parasites of the red rockfish Sebastes capensis off northern Chile. J. Parasitol. 84: 783-788. 
Gutiérrez, P. A. y S. R. Martorelli. 1994. Seasonality, distribution, and preferences sites of Demidospermus valenciennes Gutierrezet Suriano, 1992 (Monogenea: Ancyrocephalidae) in catfish. Research Rev. Parasitol. 54: 259-261.

Hayward, C. J. 1997. Helminths ectoparasites of sillaginid fishes (Perciformes: Percoidei) have low species richness. Folia Parasitol. 44: 173-187.

Hayward, C. J.; K. M. Perera y K. Rohde. 1998. Assemblages of ectoparasites of a pelagic fish, slimy mackerel (Scomber australasicus), from south-eastern Australia. Int. J. Parasitol. 28: 263-273. Iannacone, J. 1990. Dinámica poblacional de la fauna parasitaria (metazoa) de Menticirrhus ophicephalus (Pisces: Sciaenidae) de la costa central peruana. Tesis Lic. en Biología. Univ. Ricardo Palma, Lima, Perú. 85 pp.

Iannacone, J. y J. L. Luque. 1994. Aspectos ecológicos de los parásitos branquiales del bagre, Galeichthyis peruanus (L.) (Pisces: Teleostei) en la Costa Central del Perú. Bol. de Lima (Perú) 16: 91 96.

Iannacone, J.; M. Reyes y L. Ayala. 1995. Patrones de distribución espacial de cuatro ectoparásitos branquiales del pez aguja Strongylura scapularis. Libro de Resúmenes del IX Congreso Peruano de Microbiología y Parasitología, Lima, Perú.

Iannacone, J.; B. Córdova; K. Malpartida; N. Salazar; J. Fuertes y C. Mari. 1996. Fauna parasitaria (Metazod) de Paralabrax humeralis "Cabrilla" (Pisces: Teleostei) en la costa central del Perú. Libro de Resúmenes del Congreso X Nacional de Biología.

Iannacone, J.; J. Fuertes; B. Córdova. 1997. Comunidad de ectoparásitos (Metazoa) de Paralonchurus peruanus "Coco" de Lima. Bol. Per, Parasitol. 12: 103.

Janovy, J. Jr; M. A. McDowell y M T. Ferding. 1991. The niche of Salsuyinus thalkeni, a gili parasite of Fundulus zebrinus. J. Parasitol. 77: 697-702.
Kingston, N.; W. A. Dillon y W. Hargis. 1969. Studies on larval monogenea of fishes from Cnesapeake Bay area, Part I. J. Parasitol. 55: 544-558.

Jara, C. 1998. Prevalencia e intensidad de parasitismo por helmintos en cuatro especies de peces de la zona norte del mar peruano. Rev. Per. Parasitol. 113: 76-83.

Lasse, B. A. 1989. Seasonal population dynamics and maturation of Neoechinorhynchus pungitius (Acanthocephala: Neoechinorhynchidae) infecting brock stickleback, Culaea inconstans from Sioux Creek, Wisconsin, USA, Can. J. Zool. 67: 590-595.

Lo, C. M. 1999. Mating rendezvous in monogenean gill parasites of the humbug Dascyllus aruanus (Pisces: Pomacentridae). J. Parasitol. 85: 1178 1180.

Luque, J. L. 1994a. Dinámica poblacional y estructura de la comunidad de metazoarios parásitos de Menticirrhus ophicephalus (Pisces: Sciaenidae) de la costa peruana. Rev. Biol. Trop. 42: 21-29.

Luque, J. L. 1994b. Dinámica poblacional de Metamicrocotyla macracantha (Monogenea: Microcotylidiii) parásito de Mugil cephalus (Pisces: Mugilidae) en la costa central peruana. Rev. Biol. Trop. 42: 733-735.

Luque, J. L. 1996. Distribución transversal y asociaciones interespecíficas en las comunidades de metazoarios ectoparásitos de peces esciênidos marinos del Perú. Rev. Biol. Trop. 44: 383.390.

Luque, J. L. y M. Oliva. 1993. Análisis cuantitativo y estructura de la comunidad parasitaria de Paralonchurus peruanus (Usteichthyes: Sciaenidae) de la costa peruana. Parasitol. al día 17: 107-111.

Luque, J. L.; J. Iannacone y C. Farfán. 1991. Parásitos de peces óseos marinos en el Perú: lista di especies conocidas. Bol. de Lima (Perú) 74: 17-28. 
Mendo, J.; M. Samamé; A. Wosnitza-Mendo; J. Mendieta y J. Castillo. 1988. Análisis biológico-pesquero y poblacional de la cachema (Cynoscion analis) del área de Paita Perú. Bol. Inst. Mar. Perú. Vol. 12: Callao, Perú.

Morand, S.; R. Poulin; K. Rohde y C. Hayward. 1999. Aggregation and species coexistence of ectoparasites of marine fishes. Int. J. Parasitol. 29: 663-672.

Oliva, M. E. 1999. Metazoan parasites of the jack mackerel Trachurus murphyi (Teleostei, Carangidae) in a latitudinal gradient from South American (Chile and Perú). Parasite 6: 223-230.

Oliva, M. E. y J. L. Luque. 1998. Metazoan parasites infracommunities in five sciaenids from the Central Peruvian Coast. Mem. Inst. Oswaldo Cruz 93: 175-180.

Oliva, M. E.; J. L. Luque y J. Iannacone. 1989. Prevalencia y patrones de distribución de tres especies de monogeneos en las branquias de Siellifer minor (Tschudi, 1844) (Osteichthyes: Sciaenidae). Rev. Ibér. Partasitol. 49: 209-2l 4.

Oliva, M. E.; J. L. Luque y J. Iannacone. 1990. The metazoan parasites of Stellifer minor (Tschudi, 1844): An ecological approach. Mem. Inst. Oswaldo Cruz 85 : 271-274.

Oliver, G. 1982. Quelques aspects de la spécificite parasitaire chez les Diplectanidae Bychowsky, 1957 (Monogenea, Monopisthocotylea). Mém. Mus. Nat. D’hist. Natur. (Sér. Zool.). 123: 295-301.

Rohde, K. 1980. Comparative studies on microhabitat utilization by ectoparasites of some marine fishes from the North Sea and Papua New Guinea. Zool. An?. Jena 204: 27-63.

Rohde, K. 1982. Ecology of Marine parasites. University of Queensland, Quensland, Australia. $245 \mathrm{pp}$.

Rohde, K. 1991. Intra- and interspecific interactions in low density populations in resource-rich habitats. Oikos 60:91104.

Rohde, K.; C. Hayward; M. Heap y D. Gosper. 1994. A tropical assemblage of ectoparasites: gill and head parasites of Lethrinus miniatus (Teleostei: Lethrinidae). Int. J, Parasitol. 24: 1031 1053.

Rohde, K.; C. Hayward y M. Heap. 1995. Aspects of the ecology of metazoan ectoparasites of marine fishes. Int. J. Parasitol. 25: 945-970.

Saldarriaga, C. 1977. La parasitosis y su relación con el factor de condición, sexo y la longitud de la "cachema" Cynoscion analis. Tesis $\mathrm{Br}$. CC, BB. Univer. Nacional de Trujillo. Trujillo. Perú.

Sasal, P.; N. Niquil y P. Bartoli. 1999. Community structure of digenean parasites of sparid and labrid fishes of the Mediterranean sea: a new approach. Parasitology 119: 635-648.

Silan, P. y C. Maillard. 1989. Biologie comparée du développement et discrimination des Diplectanidae ectoparásites du Bar (Teleostei). Ann. Sci. Nat. Zool. Biol. Anim. 10: 31-45.

Sutherland, D. R. 1989. Seasonal distribution and ecology of three helminths species infecting carp (Cyprinus carpio) in Northwest Iowa, USA. Can. J. Zool. 67. 692-698.

Tarazona, J. y S. Valle. 1998. La diversidad biol6gica en el mar peruano. Halfter, G. (comp.). En: La Diversidad Biológica de Iberoamérica III. Volumen Especial, Acta Zoológica Mexicana, nueva serie. pp. 103-115. Instituto de Ecología, A. C. Xalapa, México.

Whittington, I. A. 1997. Reproduction and host-location among the parasitic Platyhelminthes. Int. J. Parasitol. 27: 705-714.

Zar, J. H. 1996. Biostatistical Análisis. Er. Ed. Prentice-Hall, Inc. Upper Saddle River. New Jersey. 662 pp. 